\title{
SOME HETEROLEPTIC COMPOUNDS OF ALUMINIUM(III) CONTAINING ACETYLACETONATO AND BRIDGED GLYCOLATO MOIETIES, $\left[\left(\mathrm{CH}_{3} \mathrm{COCHCOCH}_{3}\right)_{2} \mathrm{Al}(\mu-\mathrm{O}-\mathrm{G}-\mathrm{O}) \mathrm{Al}(\mathrm{O}-\mathrm{G}-\mathrm{O})\right]$
}

\author{
Shweta Nagar, R. Bohra* and R. C. Mehrotra \\ Department of Chemistry, University of Rajasthan, Jaipur-302 004, India <rkbohra@satyam.net.in>
}

Abstract

Some new heteroleptic bridged glycolate complexes of aluminium(III) with the composition, $\left[\left(\mathrm{CH}_{3} \mathrm{COCHCOCH}\right)_{2} \mathrm{Al}(\mu-\mathrm{O}-\mathrm{G}-\mathrm{O}) \mathrm{AI}(\mathrm{O}-\mathrm{G}-\mathrm{O})\right]$ have been prepared by the reactions of $\left[\left(\mathrm{CH}_{3} \mathrm{COCHCOCH}_{3}\right)_{2} \mathrm{Al}\left(\mu-\mathrm{OPr}^{\prime}\right)_{2} \mathrm{Al}\left(\mathrm{OPr}^{\prime}\right)_{2}\right]$ with glycols, $(\mathrm{HO}-\mathrm{G}-\mathrm{OH})$ (where $\mathrm{G}=-\left(\mathrm{CH}_{2}\right)_{2}-1 ;-\mathrm{CH}_{2} \mathrm{CH}\left(\mathrm{CH}_{3}\right)-$ 2; - $\left(\mathrm{CH}_{2}\right)_{3}-3 ;-\mathrm{CH}\left(\mathrm{CH}_{3}\right) \mathrm{CH}\left(\mathrm{CH}_{3}\right)-4 ;-\mathrm{C}\left(\mathrm{CH}_{3}\right)_{2} \mathrm{CH}_{2} \mathrm{CH}\left(\mathrm{CH}_{3}\right)-5 ;-\mathrm{C}\left(\mathrm{CH}_{3}\right)_{2} \mathrm{C}\left(\mathrm{CH}_{3}\right)_{2}-6$ and $\left.-\left(\mathrm{CH}_{2}\right)_{6^{-}} 7\right]$ in $1: 2$ molar ratio. All these compounds are soluble in common organic solvents, except the hexamethylene glycol derivative, which is partially soluble in benzene and chloroform. They show sharp melting points. Molecular weight determinations of these derivatives reveal their dimeric nature in chloroform. A plausible structure has been proposed on the basis of elemental analyses, molecular weight measurements, IR and NMR $\left({ }^{1} \mathrm{H},{ }^{13} \mathrm{C}\right.$ and ${ }^{27} \mathrm{Al}$ ) spectral studies. ${ }^{27} \mathrm{Al}$ NMR spectra show the presence of four- and six-coordinated aluminium sites. Thermogravimetric studies of 6 show a fast decomposition in the $130-325^{\circ} \mathrm{C}$ range followed by the formation of $\mathrm{Al}_{2} \mathrm{O}_{3}$ above $625^{\circ} \mathrm{C}$.

\section{Introduction}

Interest in the chemistry of metal/heterometal alkoxides have been growing continuously ${ }^{1}$ in view of their solubility in common organic solvents, oligomeric nature as well as interesting structural ${ }^{2.3}$ and bonding patterns. Metal alkoxides can be easily hydrolysed and undergo facile reactions with a variety of organic protic ligands, yielding novel products with volatile side products, which can be easily removed. It is the property that has been increasingly exploited not only to utilize them as unique synthons for mixed ligand/mixed metal species ${ }^{4-9}$ but also for the low temperature preparation of novel oxide ceramic materials.

Our recent efforts, therefore, have been focussed on the synthesis and characterisation of heteroleptic derivatives of aluminium(III) where the labile alkoxy groups in $\left[\mathrm{Al}(\mathrm{OR})_{3}\right]_{\mathrm{n}}$ are replaced by various ligands. ${ }^{10-16}$ It has been observed that steric and electronic factors also play a very important role in mixed aluminium(alkoxide) $\beta$-diketonate chemistry. ${ }^{2,12}$ For example, compounds of the type $\left[(\beta-\mathrm{dik} .)_{2} \mathrm{Al}(\mu\right.$ $\mathrm{OR})_{2} \mathrm{Al}(\mathrm{OR})_{2}$ ] are discrete unsymmetrical dimers ${ }^{2}$, both in solution and in the solid state due to the presence of comparatively quite stable four- and six-coordinated aluminium rather than a symmetric structure of the type $\left[(\beta\right.$-dik. $)(\mathrm{OR}) \mathrm{Al}(\mu-\mathrm{OR})_{2} \mathrm{Al}(\mathrm{OR})(\beta$-dik. $\left.)\right]$, having penta-coordinated aluminium(III).

In view of the above, in this paper, we report the preparation and characterisation of some novel bridged glycolate compounds of aluminium(III) by the reactions of $\left[\left(\mathrm{CH}_{3} \mathrm{COCHCOCH}_{3}\right)_{2} \mathrm{Al}(\mu-\right.$ $\left.\left.\mathrm{OPr}^{\mathrm{l}}\right)_{2} \mathrm{Al}\left(\mathrm{OPr}^{\prime}\right)_{2}\right]$ with glycols in 1:2 molar ratio.

\section{Materials and Methods}

All the chemicals used were of reagent grade. Moisture is carefully excluded throughout experimental manipulations. Aluminium isopropoxide was prepared by a reported method ${ }^{17}$. Glycols and acetylacetone were distilled before use. Benzene, isopropyl alcohol, chloroform and toluene were carefully dried by usual methods ${ }^{18}$. Aluminium was estimated as oxinate gravimetrically ${ }^{19}$. Isopropyl alcohol in the azeotrope was estimated oxidimetrically ${ }^{20}$ using $\mathrm{IN} \mathrm{K}_{2} \mathrm{Cr}_{2} \mathrm{O}_{7}$ solution in $12.5 \% \mathrm{H}_{2} \mathrm{SO}_{4}$ acid.The starting material $\left[\left(\mathrm{CH}_{3} \mathrm{COCHCOCH}\right)_{2} \mathrm{AI}\left(\mu-\mathrm{OPr}^{5}\right)_{2} \mathrm{Al}\left(\mathrm{OPr}^{1}\right)_{2}\right]$ was prepared by reported method ${ }^{16-18}$.

Infrared spectra were recorded as Nujol mulls on a Nicolet Magna 550 spectrophotometer in the range 4000-400 $\mathrm{cm}^{-1}$. ${ }^{1} \mathrm{H}$ NMR spectra were recorded on a Jeol FX 900 spectrometer in $\mathrm{CDCl}_{3}$ using TMS as an internal reference. ${ }^{13} \mathrm{C}$ NMR spectral studies have been carried out in chloroform at $22.49 \mathrm{MHz}$ using $\mathrm{D}_{2} \mathrm{O}$ locks. ${ }^{27} \mathrm{Al}$ NMR spectral studies have been carried out in toluene using aluminium nitrate as a standard reference. Molecular weight measurements were carried out on a Knauer Vapour Pressure Osmometer (in $\mathrm{CHCl}_{3}$ ) at $45^{\circ} \mathrm{C}$ temperature. $\mathrm{C}$ and $\mathrm{H}$ were analyzed on a Perkin Elmer $\mathrm{C}, \mathrm{H}, \mathrm{N}$ and $\mathrm{S}$ II series 2400 analyser. Thermograms were recorded on a thermogravimetric analyzer system at Seiko instrument Inc. at a heating rate of $10^{\circ} \mathrm{C} \mathrm{min} \min ^{-1}$ in a static air atmosphere using $\mathrm{Al}_{2} \mathrm{O}_{3}$ as reference material.

All these mixed ligand aluminium(III) compounds were synthesized by a similar route and hence, the synthesis of only one representative compound is described below. Details of the synthesis of other derivatives and the analytical data have been summarized in Table I.

Preparation of [ $\left.\left(\mathrm{CH}_{3} \mathrm{COCHCOCH}\right)_{2} \mathrm{Al}\left(\mathrm{O}-\left(\mathrm{CH}_{2}\right)_{2}-\mathrm{O}\right\} \mathrm{Al}\left(\mathrm{O}_{-}\left(\mathrm{CH}_{2}\right)_{2}-\mathrm{O}\right\}\right]$

Ethylene glycol $(0.514 \mathrm{~g}, 8.29 \mathrm{mmol})$ was added in benzene solution $(\sim 40 \mathrm{ml})$ of $\left[\left(\mathrm{CH}_{3} \mathrm{COCHCOCH}_{3}\right)_{2} \mathrm{AI}\left(\mu-\mathrm{OPr}^{\prime}\right)_{2} \mathrm{Al}\left(\mathrm{OPr}^{\prime}\right)_{2}\right](2.02 \mathrm{~g}, 4.14 \mathrm{mmol})$ and was refluxed on a fractionating column for $\sim 4 \mathrm{~h}$. The isopropanol formed in the reaction was fractionated out azeotropically with benzene. Progress as 
well as the completion of the reaction was checked by the estimation of the isopropanol in the azeotrope by oxidimetry $^{20}$. The product was soluble in benzene. After stripping off the solvent under reduced pressure, a shining white solid was obtained in a quantitative yield, which was purified by recrystallization from a mixture of dichloromethane and $n$-hexane.

TABLE-I: Synthetic and analytical data of $\left.\mid \mathrm{CH}_{3} \mathrm{COCHCOCH}_{3}\right)_{2} \mathrm{Al}(\mu-\mathrm{O}-\mathrm{G}-\mathrm{O}) \mathrm{Al}(\mathrm{O}-\mathrm{G}-\mathrm{O})$

\begin{tabular}{|c|c|c|c|c|c|c|c|c|}
\hline \multirow[t]{2}{*}{$\begin{array}{c}\text { S. } \\
\text { No. }\end{array}$} & Reactants(g) & \multirow{2}{*}{$\begin{array}{l}\text { PrioH(g) } \\
\text { Found } \\
\text { (Calc.) }\end{array}$} & \multirow{2}{*}{$\begin{array}{l}\text { Compd. } \\
\text { No. } \\
\text { \%yield }\end{array}$} & \multicolumn{3}{|c|}{$\begin{array}{c}\text { Analysis \% } \\
\text { Found(Calc.) }\end{array}$} & \multirow{2}{*}{$\begin{array}{l}\text { M.Wt. } \\
\text { Found } \\
\text { (Calc.) }\end{array}$} & \multirow{2}{*}{$\begin{array}{c}\text { M.Pt } \\
{ }^{\circ} \mathbf{C}\end{array}$} \\
\hline & $\begin{array}{l}\text { a. } \\
{\left[\mathrm{Al}\left(\mathrm{CH}_{3} \mathrm{COCHCOCH}\right)_{2}\left(\mathrm{OPr}^{-}\right)\right]_{2}} \\
\text { b. } \mathrm{HO}-\mathrm{G}-\mathrm{OH}\end{array}$ & & & $\mathrm{Al}$ & C & $\mathrm{H}$ & & \\
\hline 1. & $\begin{array}{l}\text { a. } 2.02 \\
\text { b. } \mathrm{G}=-\left(\mathrm{CH}_{2}\right)_{2-}^{-} \\
\quad 0.514\end{array}$ & $\begin{array}{c}0.97 \\
(0.98)\end{array}$ & $\begin{array}{l}\text { (1) } \\
98\end{array}$ & $\begin{array}{c}14.49 \\
(14.51)\end{array}$ & $\begin{array}{c}44.75 \\
(45.16)\end{array}$ & $\begin{array}{c}5.70 \\
(5.91)\end{array}$ & $\begin{array}{c}370 \\
(372)\end{array}$ & 110 \\
\hline 2. & $\begin{array}{l}\text { a. } 2.25 \\
\text { b. } \mathrm{G}=-\mathrm{CH}_{2} \mathrm{CH}\left(\mathrm{CH}_{3}\right)- \\
0.686\end{array}$ & $\begin{array}{c}0.98 \\
(0.98)\end{array}$ & $\begin{array}{l}(2) \\
97\end{array}$ & $\begin{array}{c}13.18 \\
(13.50)\end{array}$ & $\begin{array}{c}47.50 \\
(48.00)\end{array}$ & $\begin{array}{c}6.70 \\
(6.50)\end{array}$ & $\begin{array}{c}395 \\
(400)\end{array}$ & 120 \\
\hline 3. & $\begin{array}{l}\text { a. } 1.37 \\
\text { b. } \mathrm{G}=-\left(\mathrm{CH}_{2}\right)_{3-}^{-} \\
0.429\end{array}$ & $\begin{array}{c}0.62 \\
(0.67)\end{array}$ & $\begin{array}{l}\text { (3) } \\
99\end{array}$ & $\begin{array}{c}13.33 \\
(13.50)\end{array}$ & $\begin{array}{c}47.80 \\
(48.00)\end{array}$ & $\begin{array}{c}6.60 \\
(6.50)\end{array}$ & $\begin{array}{c}397 \\
(400)\end{array}$ & 122 \\
\hline 4. & $\begin{array}{l}\text { a. } 2.52 \\
\text { b. } \mathrm{G}=-\mathrm{CH}\left(\mathrm{CH}_{3}\right) \mathrm{CH}\left(\mathrm{CH}_{3}\right)^{-} \\
0.928\end{array}$ & $\begin{array}{c}1.20 \\
(1.20)\end{array}$ & $\begin{array}{l}(4) \\
99\end{array}$ & $\begin{array}{c}12.45 \\
(12.61)\end{array}$ & $\begin{array}{c}50.12 \\
(50.40)\end{array}$ & $\begin{array}{c}6.84 \\
(7.00)\end{array}$ & $\begin{array}{c}420 \\
(428)\end{array}$ & 125 \\
\hline 5. & $\begin{array}{l}\text { a. } 2.36 \\
\text { b. } \mathrm{G}=-\mathrm{C}\left(\mathrm{CH}_{3}\right)_{2} \mathrm{CH}_{2} \mathrm{CH}\left(\mathrm{CH}_{3}\right)- \\
\quad 1.400\end{array}$ & $\begin{array}{c}1.20 \\
(1.20)\end{array}$ & $\begin{array}{l}(5) \\
100\end{array}$ & $\begin{array}{c}10.94 \\
(11.15)\end{array}$ & $\begin{array}{c}54.21 \\
(54.50)\end{array}$ & $\begin{array}{c}7.50 \\
(7.80)\end{array}$ & $\begin{array}{c}480 \\
(484)\end{array}$ & 115 \\
\hline 6. & $\begin{array}{l}\text { a. } 2.81 \\
\text { b. } \mathrm{G}=-\mathrm{C}\left(\mathrm{CH}_{3}\right)_{2} \mathrm{C}\left(\mathrm{CH}_{3}\right)_{2}- \\
1.358\end{array}$ & $\begin{array}{c}1.34 \\
(1.38)\end{array}$ & $\begin{array}{l}(6) \\
98\end{array}$ & $\begin{array}{c}10.90 \\
(11.10)\end{array}$ & $\begin{array}{c}54.30 \\
(54.50)\end{array}$ & $\begin{array}{c}7.50 \\
(7.80)\end{array}$ & $\begin{array}{c}481 \\
(484)\end{array}$ & 120 \\
\hline 7. & $\begin{array}{l}\text { a. } 2.33 \\
\text { b. } \mathrm{G}=-\left(\mathrm{CH}_{2}\right)_{6}^{-} \\
\quad 1.130\end{array}$ & $\begin{array}{c}1.09 \\
(1.10)\end{array}$ & $\begin{array}{l}\text { (7) } \\
97\end{array}$ & $\begin{array}{c}10.99 \\
(11.10)\end{array}$ & $\begin{array}{c}54.25 \\
(54.50)\end{array}$ & $\begin{array}{c}7.70 \\
(7.80)\end{array}$ & $\begin{array}{c}478 \\
(484)\end{array}$ & 128 \\
\hline
\end{tabular}

\section{Resulits and Discussion}

The reactions of $\left[\left(\mathrm{CH}_{3} \mathrm{COCHCOCH}\right)_{2} \mathrm{Al}\left(\mu-\mathrm{OPr}^{\mathrm{i}}\right)_{2} \mathrm{Al}\left(\mathrm{OPr}^{1}\right)_{2}\right]$ with glycols $(\mathrm{HO}-\mathrm{G}-\mathrm{OH})$ in a $1: 2$ molar ratio in refluxing benzene under anhydrous conditions are quite facile and four moles of isopropanol could be collected easily:

$\left[\left(\mathrm{CH}_{3} \mathrm{COCHCOCH}\right)_{2} \mathrm{Al}\left(\mu-\mathrm{OPr}^{i}\right)_{2} \mathrm{Al}\left(\mathrm{OPr}^{\mathrm{i}}\right)_{2}\right]+2 \mathrm{HO}-\mathrm{G}-\mathrm{OH} \underset{\text { Reflux }}{\stackrel{\text { Benzene }}{\longrightarrow}}$
$\left[\left(\mathrm{CH}_{3} \mathrm{COCHCOCH}_{3}\right)_{2} \mathrm{Al}(\mu-\mathrm{O}-\mathrm{G}-\mathrm{O}) \mathrm{Al}(\mathrm{O}-\mathrm{G}-\mathrm{O})\right]+4 \mathrm{Pr}^{\mathrm{i} O H} \uparrow$

(where $\mathrm{G}=\left(-\mathrm{CH}_{2}\right)_{2}-\mathrm{I} ;-\mathrm{CH}_{2} \mathrm{CH}\left(\mathrm{CH}_{3}\right)-2 ;-\left(\mathrm{CH}_{2}\right)_{3} 3 ;-\mathrm{CH}\left(\mathrm{CH}_{3}\right) \mathrm{CH}\left(\mathrm{CH}_{3}\right)-4 ;-\mathrm{C}\left(\mathrm{CH}_{3}\right)_{2} \mathrm{CH}_{2} \mathrm{CH}\left(\mathrm{CH}_{3}\right)$ 5; $-\mathrm{C}\left(\mathrm{CH}_{3}\right)_{2} \mathrm{C}\left(\mathrm{CH}_{3}\right)_{2}-6$ and $\left.-\left(\mathrm{CH}_{2}\right)-7\right)$

The progress of these reactions has been followed by estimating the isopropanol liberated azeotropically. All these derivatives are hygroscopic, white shining solids and are soluble in common organic solvents, except the hexamethylene glycol derivative that is partially soluble in benzene and chloroform. These can be purified by recrystallization from a mixture of dichloromethane and $n$-hexane $(3: 1)$. Molecular weight determinations of all these complexes by osmometry in $\mathrm{CHCl}_{3}$ indicate their dimeric nature.

\section{IR spectra}

The tentative assignments of some of the important bands have been made and are summarized in Table- II. The broad band at $3450-3500 \mathrm{~cm}^{-1}$ due to $-\mathrm{OH}$ group of the free glycols disappeared in the spectra of these derivatives, suggesting the deprotonation of the $-\mathrm{OH}$ groups and formation of $\mathrm{Al}-\mathrm{O}$ bonds. Bands in the region $645-655 \mathrm{~cm}^{-1}$ may tentatively be assigned to Al-O stretching vibrations ${ }^{14}$. The appearance of $v(C-$ O) stretching vibrations (due to terminal and bridging glycol moieties) towards lower frequencies $\left(15 \mathrm{~cm}^{-1}\right)$ further supports the formation of $\mathrm{Al}-\mathrm{O}$ bonds. In all these derivatives the absence of any band at $1720 \mathrm{~cm}^{-1}$ (due to the carbonyl stretching frequency of free acetylacetone) and the presence of strong bands in the range $1590-1610$ and $1520-1535 \mathrm{~cm}^{-1}$ due to $v(C-O)$ and $v(C-C)$ stretching vibrations ${ }^{10-14}$, respectively of the acetylacetone moiety suggest the bidentate quasiaromatic nature of the ligand. The $v(\mathrm{Al}-\mathrm{O}-\mathrm{Al})$ vibrations ${ }^{21}$ have been observed in the region $755-765 \mathrm{~cm}^{-1}$. 
TABLE-II: IR spectral data $\left(\mathrm{cm}^{-1}\right)$ of $\left.]\left(\mathrm{CH}_{3} \mathrm{COCHCOCH}_{3}\right)_{2} \mathrm{Al}(\mu-\mathrm{O}-\mathrm{G}-\mathrm{O}) \mathrm{Al}(\mathrm{O}-\mathrm{G}-\mathrm{O})\right]$

\begin{tabular}{|c|c|c|c|c|c|c|}
\hline \multirow[t]{2}{*}{$\#$} & \multirow[t]{2}{*}{ Compound } & \multicolumn{2}{|c|}{$\begin{array}{c}\begin{array}{c}\text { Acetylacetonato } \\
\text { moiety }\end{array} \\
\end{array}$} & \multicolumn{3}{|l|}{$\begin{array}{l}\text { Glycolato } \\
\text { moiety }\end{array}$} \\
\hline & & $v(C)$ & $v(C)$ & $v(C \quad O)$ & $v \mathrm{Al}-\mathrm{O}$ & $v(A|-0-A|)$ \\
\hline 1. & {$\left[\left(\mathrm{CH}_{3} \mathrm{COCHCOCH}\right)_{2} \mathrm{Al}\left\{\mu-\mathrm{O}-\left(\mathrm{CH}_{2}\right)_{2}-\mathrm{O}\right\} \mathrm{Al}\left(\mathrm{O}-\left(\mathrm{CH}_{2}\right)_{2}-\mathrm{O}\right\}\right]$} & $1520 \mathrm{~s}$ & $1605 \mathrm{~s}$ & $1045 \mathrm{~m}$ & $650 w$ & $760 w$ \\
\hline 2. & $\begin{array}{l}{\left[\left(\mathrm{CH}_{3} \mathrm{COCHCOCH}\right)_{2} \mathrm{Al}\left\{\mu-\mathrm{O}-\mathrm{CH}_{2} \mathrm{CH}\left(\mathrm{CH}_{3}\right)-\mathrm{O}\right\} \mathrm{Al}(\mathrm{O}-\right.} \\
\left.\left.\mathrm{CH}_{2} \mathrm{CH}\left(\mathrm{CH}_{3}\right) \mathrm{O}\right\}\right]\end{array}$ & $1535 \mathrm{~s}$ & $1610 \mathrm{~s}$ & $1055 \mathrm{~m}$ & $652 w$ & $765 w$ \\
\hline 3. & {$\left[\left(\mathrm{CH}_{3} \mathrm{COCH} / \mathrm{COCH}\right)_{2} \mathrm{Al}\left\{\mu-\mathrm{O}-\left(\mathrm{CH}_{2}\right)_{3}-\mathrm{O}\right\} \mathrm{Al}\left(\mathrm{O}-(\mathrm{CH},)_{3}-\mathrm{O}\right\}\right]$} & $1534 \mathrm{~s}$ & $1602 s$ & $1052 \mathrm{~m}$ & $655 w$ & $762 w$ \\
\hline 4. & $\begin{array}{l}{\left[\left(\mathrm{CH}_{3} \mathrm{COCHCOCH}\right)_{2} \mathrm{Al}\left\{\mu-\mathrm{O}-\mathrm{CH}\left(\mathrm{CH}_{3}\right) \mathrm{CH}\left(\mathrm{CH}_{3}\right) \mathrm{O}\right\}\right.} \\
\left.\mathrm{Al}\left(\mathrm{OCH}\left(\mathrm{CH}_{3}\right) \mathrm{CH}\left(\mathrm{CH}_{3}\right) \mathrm{O}\right\}\right]\end{array}$ & $1530 \mathrm{~s}$ & $1601 \mathrm{~s}$ & $1050 \mathrm{~m}$ & $645 w$ & $760 w$ \\
\hline 5. & $\begin{array}{l}{\left[\left(\mathrm{CH}_{3} \mathrm{COCHCOCH}\right)_{2} \mathrm{Al}\left\{\mu-\mathrm{O}-\mathrm{C}\left(\mathrm{CH}_{3}\right)_{2} \mathrm{CH}_{2} \mathrm{CH}\left(\mathrm{CH}_{3}\right)-\mathrm{O}\right\}\right.} \\
\mathrm{Al}\left\{\mathrm{O}-\mathrm{C}\left(\mathrm{CH}_{3}\right)_{2} \mathrm{CH}_{2} \mathrm{CH}\left(\mathrm{CH}_{3}\right) \mathrm{O}\right\} 1\end{array}$ & $1520 \mathrm{~s}$ & $1604 s$ & $1035 \mathrm{~m}$ & $648 w$ & $755 w$ \\
\hline 6. & $\begin{array}{l}{\left[\left(\mathrm{CH}_{3} \mathrm{COCHCOCH}\right)_{3}\right) \mathrm{Al}\left\{\mu-\mathrm{OC}\left(\mathrm{CH}_{3}\right)_{2} \mathrm{C}\left(\mathrm{CH}_{3}\right)_{2} \mathrm{O}\right\} \mathrm{Al}(\mathrm{O}-} \\
\left.\left.\mathrm{C}\left(\mathrm{CH}_{3}\right)_{2} \mathrm{C}\left(\mathrm{CH}_{3}\right)_{2} \mathrm{O}\right\}\right]\end{array}$ & $1535 \mathrm{~s}$ & $1600 \mathrm{~s}$ & $1045 \mathrm{~m}$ & $651 \mathrm{lw}$ & $760 w$ \\
\hline 7. & $\left.\left[\left(\mathrm{CH}_{3} \mathrm{COCHCOCH}\right)_{2}\right)_{2} \mathrm{Al}\left\{\mu-\mathrm{O}\left(\mathrm{CH}_{2}\right)_{6}-\mathrm{O}\right\} \mathrm{Al}\left\{\mathrm{O}-\left(\mathrm{CH}_{2}\right)_{6}-\mathrm{O}\right\}\right]$ & $1525 \mathrm{~s}$ & $1590 \mathrm{~s}$ & $1042 \mathrm{~m}$ & $654 w$ & $765 w$ \\
\hline
\end{tabular}

\section{${ }^{1}$ H NMR Spectra}

The important signals in the 'H NMR spectra of these derivatives are summarized in Table- III. A comparison of the spectra of the free glycols with the spectra of the corresponding derivatives show the absence of $-\mathrm{OH}$ signals, indicating deprotonation of the hydroxy group of the glycols and formation of $\mathrm{Al}-\mathrm{O}$ bond. This gets support from the significant shifting of the positions of $-\mathrm{OCH}_{2}$ and $-\mathrm{OCH}$ ligand protons. The absence of signals at $\delta 1.16 \mathrm{ppm}$ and $\delta 4.01 \mathrm{ppm}$ (due to methyl and methine protons of isopropoxy groups) indicate complete substitution of isopropoxy groups by the glycolate moieties in these derivatives. The presence of two protons resonances of the terminal and bridging glycolate groups indicates the nonequivalent nature of the terminal and bridging glycolate moieties. The acetylacetonate methyl and the methine signals appeared at $\delta 1.96-2.12 \mathrm{ppm}$ and $\delta 5.46-5.61 \mathrm{ppm}$, respectively

\section{${ }^{13}$ C NMR Spectra}

${ }^{13} \mathrm{C}$ NMR chemical shifts of these compounds are summarized in Table IV. ${ }^{13} \mathrm{C}$ NMR spectral data show the presence of the expected number of signals corresponding to the number of chemically different types of carbon atoms present in these compounds. The signals at $\delta$ 25.98:26.51, 100.74-100.92 and 191.19$191.30 \mathrm{ppm}$ are assigned to methyl, methine and carbonyl carbon of the acetylacetonate moiety, respectively. Similarly, two carbons signals for terminal and bridging glycolate groups were observed, suggesting that these are magnetically unequivalent. All these derivatives show downfield shifts of $-\mathrm{OCH}_{2}$ and $-\mathrm{OCH}$ carbon signals of bridging and terminal glycolate groups and observed in the regions $\delta$ 61.53-66.25 and 66.12-75.95, respectively suggesting their bonding to aluminium(III) atom.

\section{Thermal Decomposition}

Thermograms including DTG and DTA of a representative compound 6 is shown in Fig.l. The decomposition process appears to be fast and completed in three stages. The compound appears to be stable up to $c a .130^{\circ} \mathrm{C}$. 


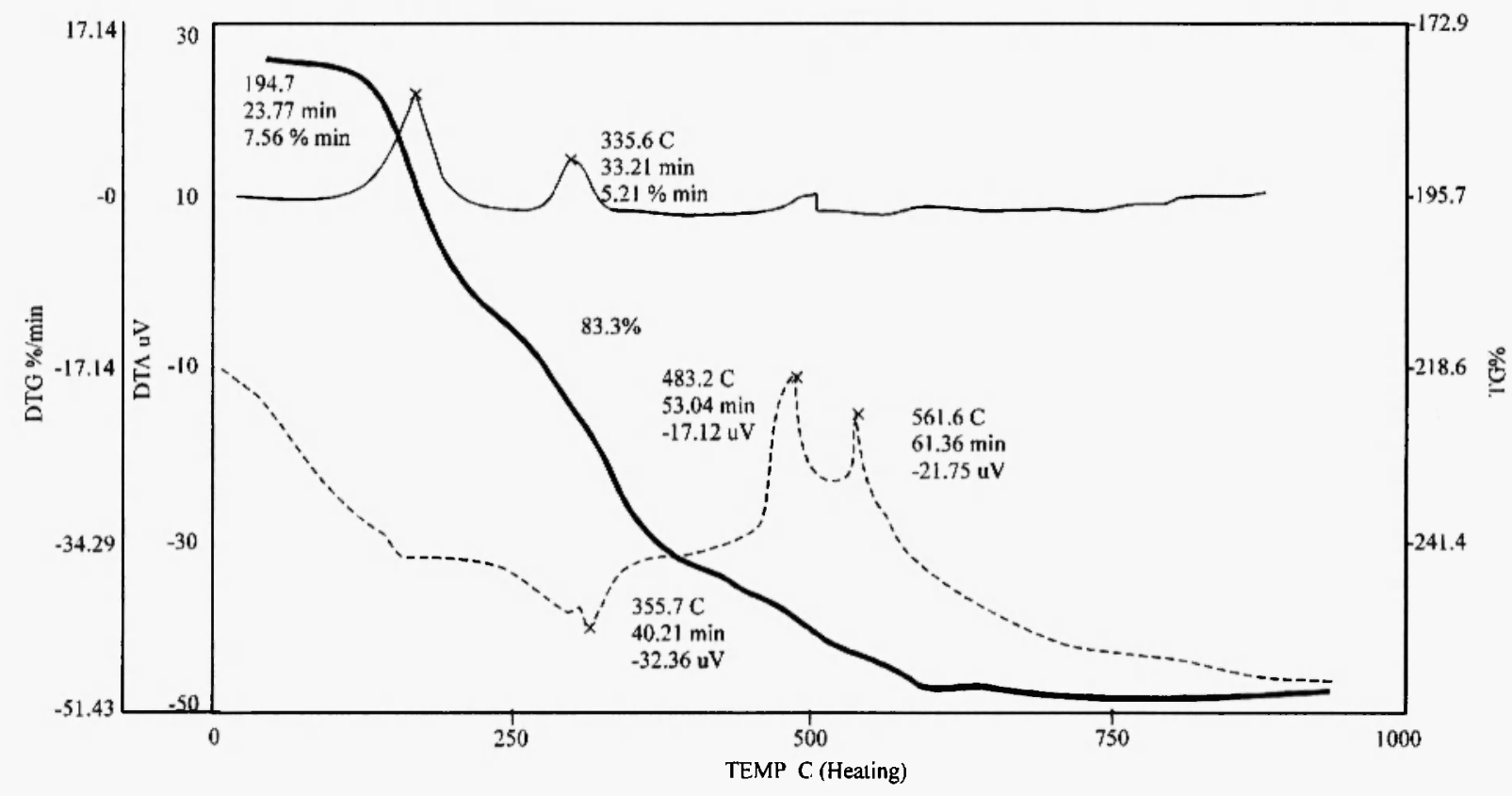

Fig. 1: Thermogram of $\left[\left(\mathrm{CH}_{3} \mathrm{COCHCOCH}\right)_{2} \mathrm{Al}\left\{\mu-\mathrm{OC}\left(\mathrm{CH}_{3}\right)_{2} \mathrm{C}\left(\mathrm{CH}_{3}\right)_{2} \mathrm{O}\right\} \mathrm{Al}\left\{\mathrm{OC}\left(\mathrm{CH}_{3}\right)_{2} \mathrm{C}\left(\mathrm{CH}_{3}\right)_{2} \mathrm{O}\right\}\right]$ Dark line TG Curve; Dotted line - DTA curve; Normal line - DTG curve

TABLE- III: ' $\mathrm{H}$ NMR spectral data $(\delta \mathrm{ppm})$ of $\left.\left[\mathrm{CH}_{3} \mathrm{COCHCOCH}_{3}\right)_{2} \mathrm{Al}(\mu-\mathrm{O}-\mathrm{G}-\mathrm{O}) \mathrm{Al}(\mathrm{O}-\mathrm{G}-\mathrm{O})\right]$

\begin{tabular}{|c|c|c|c|c|c|c|}
\hline \multirow[t]{2}{*}{$\#$} & \multirow[t]{2}{*}{ Compound } & \multicolumn{2}{|c|}{$\begin{array}{c}\text { Acetylacetanato } \\
\text { moiety }\end{array}$} & \multicolumn{2}{|c|}{ Glycolato moiety } & \multirow[t]{2}{*}{ Other protons } \\
\hline & & $-\mathrm{CH}_{3}$ & $-\mathrm{CH}<$ & $-\mathrm{OCH}_{2}$ & $-\mathrm{OCH}<$ & \\
\hline 1. & {$\left[\left(\mathrm{CH}_{3} \mathrm{COCHCOCH}\right)_{2} \mathrm{Al}\left\{\mu-\mathrm{O}-\left(\mathrm{CH}_{2}\right)_{2}-\mathrm{O}\right\} \mathrm{Al}\left(\mathrm{O}-\left(\mathrm{CH}_{2}\right)_{2}-\mathrm{O}\right\}\right]$} & $\begin{array}{l}2.00 \\
\mathrm{~s}(3 \mathrm{H})\end{array}$ & $\begin{array}{l}5.51 \\
\mathrm{~s}(4 \mathrm{H})\end{array}$ & $\begin{array}{l}3.64 \\
\mathrm{br}(4 \mathrm{H}) \\
4.12 \\
\mathrm{br}(4 \mathrm{H})\end{array}$ & - & - \\
\hline 2. & $\begin{array}{l}\mathrm{I}\left(\mathrm{CH}_{3} \mathrm{COCHCOCH}\right)_{2} \mathrm{Al}\left\{\mu-\mathrm{O}-\mathrm{CH}_{2} \mathrm{CH}\left(\mathrm{CH}_{3}\right)-\mathrm{O}\right\} \\
\left.\mathrm{Al}\left(\mathrm{O}-\mathrm{CH}_{2} \mathrm{CH}\left(\mathrm{CH}_{3}\right) \mathrm{O}\right\}\right]\end{array}$ & $\begin{array}{l}1.99 \\
\mathrm{~s}(3 \mathrm{H})\end{array}$ & $\begin{array}{l}5.61 \\
\mathrm{~s}(4 \mathrm{H})\end{array}$ & $\begin{array}{c}3.45 \\
\operatorname{br}(2 \mathrm{H}) \\
4.00 \\
\operatorname{br}(2 \mathrm{H})\end{array}$ & $\begin{array}{c}4.34 \\
\operatorname{br}(1 \mathrm{H}) \\
4.39 \\
\operatorname{br}(\mathrm{IH})\end{array}$ & $\begin{array}{l}1.64, \operatorname{br}(3 \mathrm{H}) \\
2.01, \operatorname{br}(3 \mathrm{H})\end{array}$ \\
\hline 3. & {$\left[\left(\mathrm{CH}_{3} \mathrm{COCHCOCH}\right)_{2} \mathrm{Al}\left\{\mu-\mathrm{O}-\left(\mathrm{CH}_{2}\right)_{3}-\mathrm{O}\right\} \mathrm{Al}\left(\mathrm{O}-\left(\mathrm{CH}_{2}\right)_{3}-\mathrm{O}\right\}\right]$} & $\begin{array}{l}2.06 \\
\mathrm{~s}(3 \mathrm{H})\end{array}$ & $\begin{array}{l}5.48 \\
\mathrm{~s}(\mathrm{IH})\end{array}$ & $\begin{array}{c}3.85 \\
\operatorname{br}(4 \mathrm{H}) \\
4.29 \\
\operatorname{br}(4 \mathrm{H})\end{array}$ & - & $\begin{array}{c}1.89, \operatorname{br}(2 \mathrm{H}) \\
2.19, \operatorname{br}(2 \mathrm{H})\end{array}$ \\
\hline 4. & $\begin{array}{l}{\left[\left(\mathrm{CH}_{3} \mathrm{COCHCOCH}\right)_{2} \mathrm{Al}\left\{\mu-\mathrm{O}-\mathrm{CH}\left(\mathrm{CH}_{3}\right) \mathrm{CH}\left(\mathrm{CH}_{3}\right) \mathrm{O}\right\}\right.} \\
\left.\mathrm{Al}\left(\mathrm{OCH}\left(\mathrm{CH}_{3}\right) \mathrm{CH}\left(\mathrm{CH}_{3}\right) \mathrm{O}\right\}\right]\end{array}$ & $\begin{array}{c}1.96 \\
\mathrm{~s}(3 \mathrm{H})\end{array}$ & $\begin{array}{l}5.61 \\
\mathrm{~s}(\mathrm{IH})\end{array}$ & - & $\begin{array}{c}3.48 \\
\operatorname{br}(2 \mathrm{H}) \\
4.72 \\
\operatorname{br}(2 \mathrm{H})\end{array}$ & $\begin{array}{l}1.20, \operatorname{br}((6 \mathrm{H}) \\
1.52, \operatorname{br}(6 \mathrm{H})\end{array}$ \\
\hline 5. & $\begin{array}{l}\mathrm{I}\left(\mathrm{CH}_{3} \mathrm{COCHCOCH}\right)_{2} \mathrm{Al}\left\{\mu-\mathrm{O}-\mathrm{C}\left(\mathrm{CH}_{3}\right)_{2} \mathrm{CH}_{2} \mathrm{CH}\left(\mathrm{CH}_{3}\right)-\mathrm{O}\right\} \\
\left.\mathrm{Al}\left\{\mathrm{O}-\mathrm{C}\left(\mathrm{CH}_{3}\right)_{2} \mathrm{CH}_{2} \mathrm{CH}\left(\mathrm{CH}_{3}\right) \mathrm{O}\right\}\right]\end{array}$ & $\begin{array}{l}2.00 \\
\mathrm{~s}(3 \mathrm{H})\end{array}$ & $\begin{array}{l}5.48 \\
\mathrm{~s}(\mathrm{lH})\end{array}$ & $\cdot$ & $\begin{array}{c}4.05 \\
\operatorname{br}(\mathrm{lH}) \\
4.34 \\
\operatorname{br}(\mathrm{IH})\end{array}$ & $\begin{array}{l}1.01, \mathrm{br}(6 \mathrm{H}) ; 1.20, \mathrm{br} \\
(6 \mathrm{H}) ; 1.71, \mathrm{br}(2 \mathrm{H}) \\
1.91, \mathrm{br}(2 \mathrm{H}), 2.06, \mathrm{br} \\
(3 \mathrm{H}), 2.42, \mathrm{br}(3 \mathrm{H})\end{array}$ \\
\hline 6. & $\begin{array}{l}\left(\mathrm{CH}_{3} \mathrm{COCHCOCH}\right)_{2} \mathrm{Al}\left\{\mu-\mathrm{OC}\left(\mathrm{CH}_{3}\right)_{2} \mathrm{C}\left(\mathrm{CH}_{3}\right)_{2} \mathrm{O}\right\} \\
\left.\mathrm{Al}\left(\mathrm{O}-\mathrm{C}\left(\mathrm{CH}_{3}\right)_{2} \mathrm{C}\left(\mathrm{CH}_{3}\right)_{2} \mathrm{O}\right\}\right]\end{array}$ & $\begin{array}{l}2.12 \\
\mathrm{~s}(3 \mathrm{H})\end{array}$ & $\begin{array}{l}5.46 \\
\mathrm{~s}(1 \mathrm{H})\end{array}$ & $=$ & - & $\begin{array}{l}1.16, \mathrm{~s}(12 \mathrm{H}) \\
1.20, \mathrm{~s}(12 \mathrm{H})\end{array}$ \\
\hline 7. & {$\left[\left(\mathrm{CH}_{3} \mathrm{COCHCOCH}\right)_{2} \mathrm{Al}\left\{\mu-\mathrm{O}\left(\mathrm{CH}_{2}\right)_{6}-\mathrm{O}\right\} \mathrm{Al}\left\{\mathrm{O}-\left(\mathrm{CH}_{2}\right)_{6}-\mathrm{O}\right\}\right]$} & $\begin{array}{l}2.00 \\
\mathrm{~s}(3 \mathrm{H})\end{array}$ & $\begin{array}{l}5.48 \\
\mathrm{~s}(\mathrm{IH})\end{array}$ & $\begin{array}{c}3.64 \\
\operatorname{br}(4 \mathrm{H}) \\
4.26 \\
\operatorname{br}(4 \mathrm{H})\end{array}$ & $\cdot$ & $\begin{array}{l}1.51, \mathrm{~m}(8 \mathrm{H}) \\
2.17, \mathrm{~m}(8 \mathrm{H})\end{array}$ \\
\hline
\end{tabular}




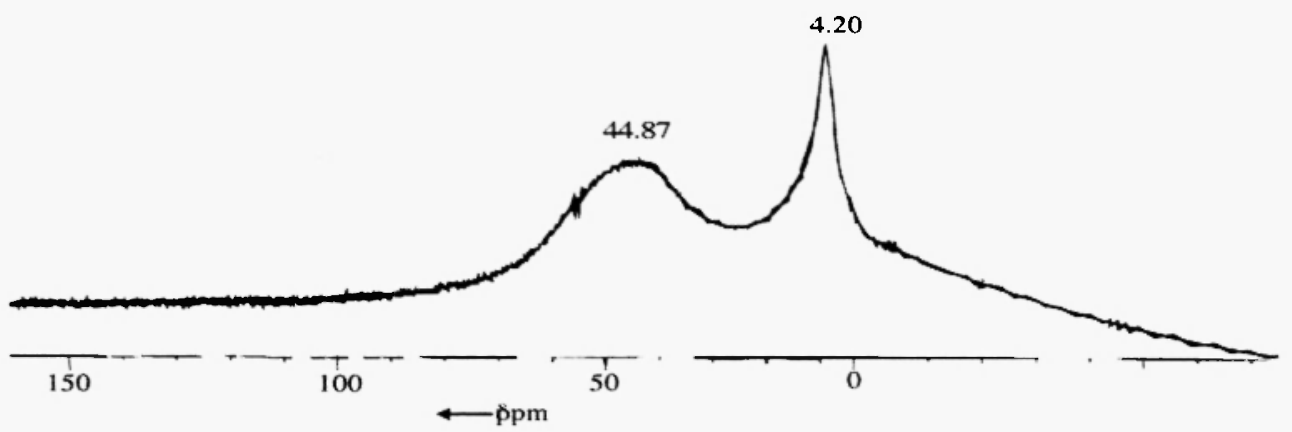

Fig.2: ${ }^{27} \mathrm{Al}$ NMR spectrum of $\left[\left(\mathrm{CH}_{3} \mathrm{COCHCOCH}\right)_{2} \mathrm{AI}\left\{\mathrm{OCC}\left(\mathrm{CH}_{3}\right)_{2} \mathrm{C}\left(\mathrm{CH}_{3}\right)_{2} \mathrm{O}\right\} \mathrm{AI}\left\{\mathrm{OC}\left(\mathrm{CH}_{3}\right)_{2} \mathrm{C}\left(\mathrm{CH}_{3}\right)_{2} \mathrm{O}\right\}\right]$

Above this temperature the decomposition starts rapidly up to $c a .325^{\circ} \mathrm{C}$ and is completed up to $625^{\circ} \mathrm{C}$. A constant weight is obtained from $625^{\circ} \mathrm{C}$ to right up to $900^{\circ} \mathrm{C}$ indicating the formation of $\mathrm{Al}_{2} \mathrm{O}_{3}$ without any organic moiety attached to aluminium. Part of the compounds seems to have been volatilized at intermediate temperatures leaving a quantity of alumina (16.7\%) less than that required by the formula weight $(21.1 \%)$. Interestingly DTA curves show one endothermic peak at $355.7^{\circ} \mathrm{C}$ and two exothermic peaks at $483^{\circ} \mathrm{C}$ and $561.6^{\circ} \mathrm{C}$ indicating the formation of intermediate products.

\section{${ }^{27}$ Al NMR Spectra}

At room temperature, the ${ }^{27} \mathrm{Al}$ NMR spectrum of a representative compound, $\left.\left[\left(\mathrm{CH}_{3} \mathrm{COCHCOCH}\right)_{2} \mathrm{Al}\left\{\mu-\mathrm{O}\left(\mathrm{CH}_{3}\right)_{2} \mathrm{C}\left(\mathrm{CH}_{3}\right)_{2} \mathrm{O}\right\}\right] \mathrm{Al}\left\{\mathrm{OC}\left(\mathrm{CH}_{3}\right)_{2} \mathrm{C}\left(\mathrm{CH}_{3}\right)_{2} \mathrm{O}\right\}\right]$, exhibits two sharp signals at $\delta$ 4.20 and $\delta 44.87$ ppm, respectively (Fig.2), suggesting the presence ${ }^{10.13 .23}$ of four- and six-coordinated aluminium(III) atoms

TABLE- IV: ${ }^{13} \mathrm{C}$ NMR spectral data $(\delta \mathrm{ppm})$ of $\left.\left.\left[\mathrm{CH}_{3} \mathrm{COCHCOCH}_{3}\right)_{2} \mathrm{Al}(\mu-\mathrm{O}-\mathrm{G}-\mathrm{O}) \mathrm{A}\right](\mathrm{O}-\mathrm{G}-\mathrm{O})\right]$

\begin{tabular}{|c|c|c|c|c|c|c|c|}
\hline \multirow{2}{*}{$\begin{array}{l}\text { S. } \\
\text { No. }\end{array}$} & \multirow[t]{2}{*}{ Compound } & \multicolumn{3}{|c|}{ Acetvlacetonato moiety } & \multicolumn{2}{|c|}{ Glycolato moiety } & \multirow{2}{*}{$\begin{array}{l}\text { Other } \\
\text { carbons }\end{array}$} \\
\hline & & $\mathrm{CH}_{3}$ & $-\mathbf{C H}$ & $>\mathrm{C} \mathrm{O}$ & $-\mathrm{OCH}_{2}$ & $-\mathrm{OCH}<$ & \\
\hline 1. & $\begin{array}{l}{\left[\left(\mathrm{CH}_{3} \mathrm{COCHCOCH}\right)_{2} \mathrm{Al}\left\{\mu-\mathrm{O}-\left(\mathrm{CH}_{2}\right)_{2}-\mathrm{O}\right\}\right.} \\
\left.\mathrm{Al}\left(\mathrm{O}-\left(\mathrm{CH}_{2}\right)_{2}-\mathrm{O}\right\}\right]\end{array}$ & 26.40 & 100.90 & 191.20 & $\begin{array}{l}62.32 \\
62.98\end{array}$ & - & - \\
\hline 2. & $\begin{array}{l}{\left[\left(\mathrm{CH}_{3} \mathrm{COCHCOCH}\right)_{2} \mathrm{Al}\left\{\mu-\mathrm{O}-\mathrm{CH}_{2} \mathrm{CH}\left(\mathrm{CH}_{3}\right)-\mathrm{O}\right\}\right.} \\
\left.\mathrm{Al}\left(\mathrm{O}-\mathrm{CH}_{2} \mathrm{CH}\left(\mathrm{CH}_{3}\right) \mathrm{O}\right\}\right]\end{array}$ & 26.43 & 100.76 & 191.21 & $\begin{array}{l}65.21 \\
66.25\end{array}$ & $\begin{array}{l}66.12 \\
68.02\end{array}$ & $18.76,18.78$ \\
\hline 3. & $\begin{array}{l}{\left[\left(\mathrm{CH}_{3} \mathrm{COCHCOCH}\right)_{2} \mathrm{Al}\left\{\mu-\mathrm{O}-\left(\mathrm{CH}_{2}\right)_{3}-\mathrm{O}\right\}\right.} \\
\left.\mathrm{Al}\left(\mathrm{O}-\left(\mathrm{CH}_{2}\right)_{3}-\mathrm{O}\right\}\right]\end{array}$ & 25.98 & 100.80 & 191.30 & $\begin{array}{l}61.53 \\
63.01\end{array}$ & - & $23.42,26.35$ \\
\hline 4. & $\begin{array}{l}{\left[\left(\mathrm{CH}_{3} \mathrm{COCHCOCH}\right)_{2} \mathrm{Al}\left\{\mu-\mathrm{O}-\mathrm{CH}\left(\mathrm{CH}_{3}\right) \mathrm{CH}\left(\mathrm{CH}_{3}\right) \mathrm{O}\right\}\right.} \\
\left.\mathrm{Al}\left(\mathrm{OCH}\left(\mathrm{CH}_{3}\right) \mathrm{CH}\left(\mathrm{CH}_{3}\right) \mathrm{O}\right\}\right]\end{array}$ & 26.43 & 100.92 & 191.29 & $=$ & $\begin{array}{l}71.45 . \\
75.95\end{array}$ & 24.32.26.33, \\
\hline 5. & $\begin{array}{l}{\left[\left(\mathrm{CH}_{3} \mathrm{COCHCOCH}\right)_{2} \mathrm{Al}\left\{\mu-\mathrm{O}-\mathrm{C}\left(\mathrm{CH}_{3}\right)_{2}\right.\right.} \\
\left.\left.\mathrm{CH}_{2} \mathrm{CH}\left(\mathrm{CH}_{3}\right)-\mathrm{O}\right\} \mathrm{Al}\left\{\mathrm{O}-\mathrm{C}\left(\mathrm{CH}_{3}\right)_{2} \mathrm{CH}_{2} \mathrm{CH}\left(\mathrm{CH}_{3}\right) \mathrm{O}\right\}\right]\end{array}$ & 26.51 & 100.83 & 191.26 & - & $\begin{array}{l}69.62 \\
70.01\end{array}$ & $\begin{array}{c}23.61,24.32 \\
25.08,26.17 \\
27.18,27.92 \\
49.92,50.72 \\
70.01,71.47\end{array}$ \\
\hline 6. & $\begin{array}{l}{\left[\left(\mathrm{CH}_{3} \mathrm{COCHCOCH}\right)_{2} \mathrm{Al}\left\{\mu-\mathrm{OC}\left(\mathrm{CH}_{3}\right)_{2} \mathrm{C}\left(\mathrm{CH}_{3}\right)_{2} \mathrm{O}\right\}\right.} \\
\left.\mathrm{Al}\left(\mathrm{O}-\mathrm{C}\left(\mathrm{CH}_{3}\right)_{2} \mathrm{C}\left(\mathrm{CH}_{3}\right)_{2} \mathrm{O}\right\}\right]\end{array}$ & 26.43 & 100.74 & 191.19 & - & $=$ & $\begin{array}{l}23.50,24.76 \\
27.26,27.85 \\
71.32,72.39\end{array}$ \\
\hline$* 7$. & $\begin{array}{l}{\left[\left(\mathrm{CH}_{3} \mathrm{COCHCOCH}\right)_{2} \mathrm{Al}\left\{\mu-\mathrm{O}\left(\mathrm{CH}_{2}\right)_{6}-\mathrm{O}\right\}\right.} \\
\left.\mathrm{Al}\left\{\mathrm{O}-\left(\mathrm{CH}_{2}\right)_{6}-\mathrm{O}\right\}\right]\end{array}$ & - & - & - & - & - & - \\
\hline
\end{tabular}

${ }^{*}{ }^{13} \mathrm{C}$ NMR spectrum could not be determined because of its less solubility

Conclusion: 
Although it is difficult to comment on the structural aspects of these derivatives without single crystal X-ray diffraction study of at least one of the products, yet on the basis of the above studies the following tentative structure may be proposed for these derivatives (Fig.3).

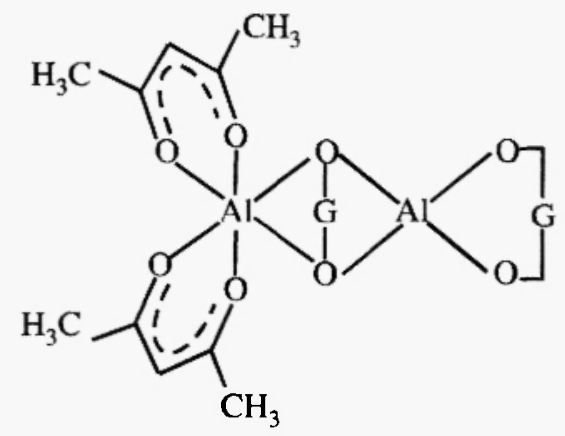

Fig.3: Proposed structure of $\left[\left(\mathrm{CH}_{3} \mathrm{COCHCOCH}_{3}\right)_{2} \mathrm{Al}\{\mu-\mathrm{O}-\mathrm{G}-\mathrm{O}\} \mathrm{Al}\{\mathrm{O}-\mathrm{G}-\mathrm{O}\}\right]$ (where $\mathrm{G}=-\left(\mathrm{CH}_{2}\right)_{2}-\mathbf{1}$; -

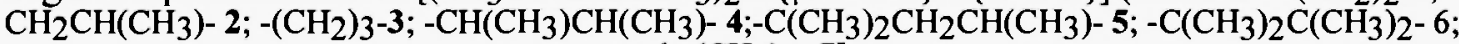
and $-\left(\mathrm{CH}_{2}\right)_{6-7]}$

\section{Acknowledgement:}

We are grateful to UGC, DST, New Delhi and D.A.E., Mumbai for financial support. One of us (S.N.) thanks the CSIR, New Delhi for the award of SRF. Prof. Mehrotra is grateful to INSA as a Senior Scientist.

\section{References:}

1. D.C.Bradley,R.C.Mehrotra, A.Singh, and I.P.Rothwell, Alkoxo and Aryloxo Derivatives of Metals. Academic Press, London (2001).

2. M.F.Garbauskas, Acta Cryst., Sect.C.Cryst.Struct.Commun., C40, 1536(1984).

3. J.H.Wengrovius, M.F.Garbauskas, E.A.Williams, R.C.Going, P.E.Donahue, J.F.Smith, J.Am.Chem.Soc., 108, 982(1986).

4. V.G. Kessler, L.G.Hubert-PFalzgraf, S. Halut, and J.C. Daran, J.Chem.Soc.Chem.Commun., 705(1994).

5. M.Verdenelli, S.Parola, L.G.Hubert-Pfalzgraf and S.Lecocq, Polyhedron, 19, 2069(2000).

6. D.W. Stephen and T.T. Nadasdi, Coord.Chem.Rev., 147, 147(1996).

7. L.B. Archer, M.J.H. Smith, and E.N. Duester, Polyhedron, 15, 929(1996).

8. L.R. Sita, R. Xi, G.P.A. Yap, L. M. Liable-Sands, and A.O. Rheingold, J.Am.Chem.Soc., 119, 756(1997).

9. $\quad$ S. Parola, R. Papiernik, J.Chem.Soc.Dalton Trans., 4631(1997).

10. A. Dhammani, R. Bohra, and R.C. Mehrotra, Main Group Met. Chem., 18(12), 687(1995).

11. A. Dhammani, R. Bohra, and R.C. Mehrotra, Polyhedron. 14 (6), 733(1995).

12. S. Nagar, A. Dhammani, R. Bohra, and R.C. Mehrotra, J. Coord. Chem., in press( 2001).

13. A. Dhammani, R.Bohra, and R.C. Mehrotra, Polyhedron., 15 (4), 733(1996).

14. R.Bohra, A.Dhammani, R.K.Sharma and R.C.Mehrotra, Synth.React.Inorg.Met.Org.Chem.,31, 681(2001).

15. N.Sharma, R.K.Sharma and R.Bohra, Main Group Met.Chem., 24,11(2002.

16. N.Sharma, R.K.Sharma, R.Bohra, J.E.Drake, M.B.Hursthouse and M.E.Light, J.Chem.Soc,,Dalton Trans, in press(2002).

17. R.C. Mehrotra, J.Indian Chem.Soc., 30, 585(1953).

18. Anita. Dhammani, Ph.D.Thesis, University of Rajasthan, Jaipur (1996).

19. A.I. Vogel, A Text Book of Quantitative Inorganic Analysis, ELBS Longmon, London (1989).

20. D.C. Bradley, F.M.A. Halim, and W. Wardlaw, J.Chem.Soc., 3450(1950).

21. R. Jain, A.K. Rai, and R.C. Mehrotra, Inorg.Chim.Acta., 126, 99(1987).

22. A. Singh, A.K. Rai and R.C. Mehrotra, Indian J.Chem., 11, 478(1973).

23. G.J. Gainsford, T. Kemmitt, and N.B. Milestone, Inorg.Chem., 34, 5244(1995).

Received: February 22, 2002 - Accepted: February 26, 2002 Accepted in publishable format: February 28, 2002 